\title{
Caracterización mineralógica de la fracción arcillosa de vertisols de la parcela de la Universidad Nacional de Piura, San Lorenzo, Piura
}

\author{
Fabian E. Nima Ramírez ${ }^{1,2}$, Jorge A. Bravo Cabrejos², Mirian E. Mejia Santillán² y Denis Silva \\ Valdiviezo $^{3}$ \\ ${ }^{1}$ Facultad de Ciencias, Universidad Nacional de Piura, Piura, Perú \\ ${ }^{2}$ Laboratorio de Análisis de Suelos, Facultad de Ciencias Físicas, Universidad Nacional Mayor de San \\ Marcos, Lima, Perú \\ ${ }^{3}$ Facultad de Agronomía, Universidad Nacional de Piura, Piura, Perú
}

Recibido 30 marzo 2011 - Aceptado 30 julio 2011

En este trabajo se estudia la caracterización mineralógica de la fracción arcillosa de dos muestras de suelos agrícolas pertenecientes a vertisols provenientes del Valle de San Lorenzo, Región Piura, mediante las técnicas analíticas de fluorescencia de rayos X por dispersión de energía (FRX), difractometría de rayos X (DRX) y espectroscopia Mössbauer (EM). Previo a su caracterización mineralógica las muestras fueron sometidas a análisis fisicoquímicos tales como medición de pH, eliminación de materia orgánica y análisis textural. En este trabajo se han utilizado dos muestras, las cuales han sido denominadas M1 y M2, y extraídas de dos calicatas diferentes a una profundidad de $50 \mathrm{~cm}$ y $150 \mathrm{~cm}$, respectivamente. Los espectros Mössbauer fueron tomados a temperatura de ambiente por la modalidad de transmisión.

Los resultados del análisis por la FRX indican que ambas muestras tienen aproximadamente la misma concentración de Fe, que fue de aproximadamente $3.85 \%$ y $3.99 \%$ para M1 y M2, respectivamente. Así mismo, se observa la presencia de algunos elementos que sirven de nutrientes a las plantas como $\mathrm{Ca}, \mathrm{K}, \mathrm{Cu}, \mathrm{Zn}$ y Mn; los cuales pueden estar presentes en los minerales de arcilla como cationes interlaminares. Los análisis mineralógicos por DRX de ambas muestras dan la presencia de fases de cuarzo, albita y de minerales de arcilla como montmorillonita, illita (tipo 2:1) y caolinita (tipo 1:1). Los resultados por la EM para ambas muestras, evidencian la presencia de dos sitios de Fe ${ }^{3+}$ en estado paramagnético ocupando sitios octaédricos (cis y trans) en la montmorillonita; adicionalmente se observa la presencia de un doblete paramagnético de $\mathrm{Fe}^{3+}$, el cual ha sido asociado a sitios tetraédricos en la montmorillonita, ya que posee corrimiento isomérico y desdoblamiento cuadrupolar pequeños. Finalmente se identifica un doblete de $\mathrm{Fe}^{2+}$, el cual no ha sido asociado a ningún mineral, ya que sus parámetros se superponen a aquellos correspondientes a la montmorillonita, illita y/o caolinita. Estos resultados también indican que el hierro se encuentra principalmente distribuido dentro los sitios octaédricos de la montmorillonita de ambas muestras.

Palabras claves: Fracción arcillosa, vertisols, minerales arcillosos, espectroscopia Mössbauer, difractometría de rayos $\mathrm{X}$.

\section{Mineralogical characterization of the clay fraction of vertisols from the Universi- dad Nacional de Piura parcel, San Lorenzo, Piura}

In this paper we study the mineralogical characterization of the clay fraction of two samples of agricultural soils from Vertisols from the Valle de San Lorenzo, Piura Region, using analytical techniques such as energy dispersion X-ray fluorescence (XRF), X-ray difractometry (XRD) and transmission Mössbauer spectroscopy (TMS). Prior to their mineralogical characterization, the samples were subjected to physicochemical analysis such as measuring their $\mathrm{pH}$, removal of organic matter and textural analysis. In this work we have used two samples, which have been referred to as M1 and M2 and drawn two different calicatas at a depth of $50 \mathrm{~cm}$ and $150 \mathrm{~cm}$ respectively. Mössbauer spectra were taken at room temperature. 
Analysis of both samples by XRF indicated that both samples have approximately the same concentration of $\mathrm{Fe}$, which was approximately 3,5\% and 3,9\% for $\mathrm{M} 1$ and M2 respectively. It also notes the presence of some elements that serve as plant nutrients as $\mathrm{Ca}, \mathrm{K}, \mathrm{Cu}, \mathrm{Zn}$ and $\mathrm{Mn}$, which may be present in clay minerals as interlayer cations. The mineralogical analysis by XRD of both samples show the presence of phases of quartz, albite and clay minerals such as montmorillonite, illite (2:1 type) and kaolinite (1:1 type). The analysis by TMS of both samples showed the presence of $\mathrm{Fe}^{3+}$ paramagnetic cations occupying octahedral sites (cis and trans) in montmorillonite; additionally it is noted the presence of a paramagnetic doublet of $\mathrm{Fe}^{3+}$, which has been associated with tetrahedral sites in montmorillonite for having small isomeric shift and quadrupole splitting. Finally, a $\mathrm{Fe}^{2+}$ doublet has been observed which has not been associated with any specific mineral since its parameters superimpose with those for montmorillonite, illite and kaolinite. These results also indicate that iron is mainly distributed within the octahedral sites of montmorillonite in both samples.

Keywords: Clay fraction, vertisols, clay minerals, X ray fluorescence, transmission Mössbauer spectroscopy, X-ray diffractometry.

El presente trabajo reporta resultados obtenidos en el estudio de la fracción arcillosa de dos muestras pertenecientes a la parcela experimental de la Facultad de Agronomía de la Universidad Nacional de Piura, localizada en el Valle de San Lorenzo, Distrito de Tambo Grande, Provincia de Sullana, Región Piura, a una latitud de $4^{\circ} 45^{\prime} \mathrm{S}$, longitud $80^{\circ} 15^{\prime}$ W y a una altitud de 200 m.s.n.m. Estos terrenos tienen la particularidad de poseer suelos con alto contenido de minerales de arcilla, por lo que están catalogados dentro de la orden de los vertisols. Los cultivos que más abundan en la zona son los de mango y de limón. La baja permeabilidad combinada con la elevada concentración de minerales expandibles, como la montmorillonita, que poseen estos suelos, los hace convenientes para cultivos que requieren de retención del agua superficial.

Con el propósito de establecer una descripción cualitativa y semicuantitativa de las diferentes especies minerales presentes en la fracción arcillosa de estos suelos agrícolas, en este trabajo se estudia su mineralogía mediante las técnicas analíticas de fluorescencia de rayos $\mathrm{X}$ por dispersión de energía (FRXED), de difractometría de rayos X (DRX) y de espectroscopía Mössbauer por transmisión (EMT) haciendo uso de la transición nuclear resonante de $\gamma 14,4 \mathrm{keV}$ en el isótopo natural de ${ }^{57} \mathrm{Fe}$.

La FRXED es una técnica que se usa con el objetivo de identificar y cuantificar los elementos químicos presentes en las muestras en estudio, mientras que, la DRX es una técnica muy útil que permite identificar los minerales de buena cristalización y de alta concentración presentes en una muestra. Si la muestra está constituida por una mezcla de minerales, los minerales presentes en baja concentración pueden pasar desapercibidos. Cada mineral es caracterizado por un patrón de reflexiones de los rayos $\mathrm{X}$ que están relacionados a los planos cristalográficos y a la estructura de celda del mineral $[1,2]$.

La EMT es una técnica nuclear que utiliza la emi- sión y absorción resonante de rayos gamma por ciertos núcleos. En consecuencia es una técnica isotópicamente selectiva por cuanto depende del isótopo que se desee observar [3,4]. La EMT de ${ }^{57} \mathrm{Fe}$ permite identificar los minerales que poseen hierro como catión principal, tales como los óxidos, hidróxidos y los oxi-hidróxidos de hierro, que denominaremos simplemente óxidos, o como catión de sustitución [5]. Cabe mencionar que los minerales de arcilla pueden ser fácilmente identificados por la EMT debido a que el hierro comúnmente sustituye de manera isomórfica al $\mathrm{Si} \mathrm{y/o} \mathrm{al} \mathrm{Al} \mathrm{dentro} \mathrm{de} \mathrm{sus}$ estructuras, o puede estar presente como catión interlaminar.

Se debe entender por fracción arcillosa a la fracción granulométrica constituida por minerales cuyo tamaño de grano es menor a $2 \mu \mathrm{m}$; y por minerales de arcilla propiamente dichos, es decir, aquellos minerales que poseen la estructura laminar de los alúmino silicatos. De hecho, el primero incluye a los segundos cuando éstos cumplen el requisito de tamaño de grano. La importancia y el interés por el estudio de la fracción arcillosa de los suelos radica en la alta actividad geoquímica de los minerales que la constituyen por contener granos muy pequeños con una razón de alta proporción de área superficial a volumen.

Denominaremos a las muestras estudiadas M1 y M2; que han sido extraídas de dos calicatas diferentes a 50 $\mathrm{cm}$ y $150 \mathrm{~cm}$ de profundidad respectivamente. Como mostramos más adelante, ambas muestras son muy parecidas en su composición mineralógica.

\section{Materiales y métodos}

\section{Preparación de muestras}

Las dos muestras fueron sometidas previamente a un análisis físico-químico, como son la medida del $\mathrm{pH}$, eliminación de materia orgánica y análisis textural [6]. El valor del $\mathrm{pH}$ nos indica la concentración de iones 
de hidrógeno que posee la solución suelo; el pH influye en el suelo en varios aspectos, pero el más significativo es en la disponibilidad de nutrientes. Es decir, el pH influye en la mayor o menor cantidad de nutrientes (fósforo, potasio, hierro, cobre, boro) que hay en un suelo para que lo puedan tomar las raíces de las plantas, ya que controla la solubilidad en agua de dichos nutrientes. Por otro lado, es importante eliminar la materia orgánica porque su presencia impide una buena resolución de los picos de difracción de rayos X, por cuanto impide el alineamiento de los granos pequeños de los minerales de arcilla, y un incremento en la reflexión de fondo en los difractogramas, además de afectar las propiedades físicas de los suelos. El análisis textural se realizó mediante el método del hidrómetro, que permitió determinar las fracciones granulométricas de arena, limo y arcilla, y de paso extraer la fracción arcillosa que contenían las muestras estudiadas. La clase textural de la muestra se deduce de la aplicación del triángulo textural de acuerdo a las normas del Departamento de Agricultura de los Estados Unidos de América [7].

\section{Medidas por FRXED}

Para obtener los espectros de FRXED se utilizó el equipo portátil de FRX marca AmpTek, cuya fuente de rayos X utiliza un blanco de plata $(\mathrm{Z}=47)$ operado a $25 \mathrm{keV}$, y un detector de estado sólido. También se utilizó un colimador de $2 \mathrm{~mm}$ de diámetro interno para el haz de rayos X incidente. La calibración en energía se realizó con una fuente radioactiva de ${ }^{57} \mathrm{Co}$. Para el análisis cuantitativo se utilizó un programa que simula los espectros utilizando el método de parámetros fundamentales ${ }^{1}$. Para el montaje de las muestras en el portamuestra de FRX se necesitó hacer pastillas circulares de ambas muestras.

\section{Medidas por DRX}

En cuanto a la adquisición de los difractogramas, en este trabajo se utilizó el difractómetro de la Facultad de Ciencias Físicas de la Universidad Nacional Mayor de San Marcos marca RIGAKU, modelo MINIFLEX, con radiación de $\mathrm{Cu}-\mathrm{K}_{\alpha}(30 \mathrm{kV}, 15 \mathrm{~mA})$, con un goniómetro vertical y con monocromador. Las muestras en bruto y sus fracciones arcillosas, molidas y previamente tamizadas en malla de $90 \mu \mathrm{m}$, fueron sometidas a un barrido $2 \theta$ desde $5^{\circ}$ hasta 650 usando un paso $2 \theta$ de $0.03^{\circ}$ / paso y un tiempo de conteo de 2 segundos por paso. Se emplearon tres métodos en la preparación de los analitos: el de polvo, de Glass Slide y de filtración al vacío (método Millipore). El segundo de estos métodos proporciona una mejor orientación de los planos

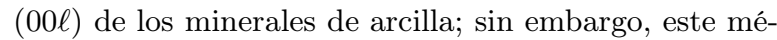

\footnotetext{
${ }^{1}$ Codificado por uno de los autores, J. Bravo Cabrejos.
}

todo se obtiene mediante un proceso de sedimentación, de manera que los minerales (como el cuarzo) con mayor tamaño de grano quedan al fondo de la muestra, produciéndose una disminución en la intensidad de sus picos de difracción. Por su lado, el método Millipore está diseñado para obtener una distribución uniforme de tamaño de grano en la muestra y una mejor orientación de los cristalitos de arcilla, comparados al primer

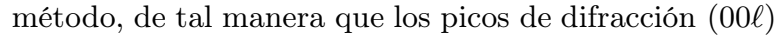
incrementen su intensidad. El análisis de los diffractograms se llevó a cabo con los programas OriginPro 7.0 y Crystallographica Search-Match.

\section{Medidas por EMT}

Los espectros Mössbauer fueron tomados por la modalidad de transmisión empleando un espectrómetro convencional. Se empleó una fuente radioactiva de ${ }^{57} \mathrm{Co}$ en una matriz de rhodio con una actividad promedio de $5 \mathrm{mCi}$. Las mediciones y análisis se llevaron a cabo en el Laboratorio de Espectroscopía Mössbauer de la Universidad Nacional Mayor de San Marcos. Los espectros fueron ajustados haciendo uso del programa NORMOS de R. A. Brand [8] en su versión para sitios cristalinos. Las muestras fueron colocadas en un portamuestra circular de $1.7 \mathrm{~cm}$ de diámetro interior, el cual fue colocado a la mitad de la distancia fuente-detector a temperatura ambiente. De acuerdo al área del portamuestra se requirieron $250 \mathrm{mg}$ de muestra en polvo, tanto para las muestras tratadas como para las muestras sin tratar. Para el almacenamiento de los espectros se utilizaron 1024 canales.

\section{Resultados y Discusión}

\section{Análisis físico-químico}

La tabla 1 muestra los resultados de los análisis de textura de las dos muestras estudiadas. De estos resultados se deduce la clase textural a la que pertenece cada muestra de suelo estudiada; la muestra M1 proviene de un suelo franco y la muestra M2 proviene de un suelo franco arcilloso arenoso. La Tabla 2 muestra los resultados de las mediciones de $\mathrm{pH}$, que indican que estos suelos son casi neutros, M1 es ligeramente alcalina y M2 es neutra.

\begin{tabular}{cccc}
\hline Muestra & Arena (\%) & Limo (\%) & Arcilla (\%) \\
\hline M1 & 43.5 & 39.2 & 17.3 \\
M2 & 54.2 & 23.1 & 22.7 \\
\hline
\end{tabular}

Tabla 1: Texturas de las muestras de San Lorenzo sin tratar. 


\begin{tabular}{cc}
\hline Muestra & $\mathrm{pH}$ \\
\hline M1 & 7.4 \\
M2 & 7.0
\end{tabular}

Tabla 2: Medición del pH de las muestras de San Lorenzo sin tratar.

\section{Análisis por Fluorescencia de Rayos X de Energía Dispersiva}

\section{Resultados cualitativos}

Los espectros de fluorescencia de las muestras M1 y M2, mostrados en las figuras 1 y 2 , respectivamente; evidencian la presencia de elementos químicos como silicio $(\mathrm{Si})$, cloro $(\mathrm{Cl})$, potasio $(\mathrm{K})$, calcio $(\mathrm{Ca})$, titanio (Ti) y hierro $(\mathrm{Fe})$. También se observan elementos traza como fósforo $(\mathrm{P})$, cromo $(\mathrm{Cr})$, manganeso $(\mathrm{Mn})$, cobre $(\mathrm{Cu})$, cinc $(\mathrm{Zn})$, rubidio $(\mathrm{Rb})$, estroncio $(\mathrm{Sr})$ y circonio $(\mathrm{Zr})$, y además azufre (S) sólo en el caso de M2. Todos estos elementos pueden estar formando parte de la estructura cristalina de los minerales presentes en M1 y $\mathrm{M} 2$, ya sea como cationes principales (Fe, $\mathrm{Si}$ ) o como cationes intercambiables (Cl, K, Ca, etc.).

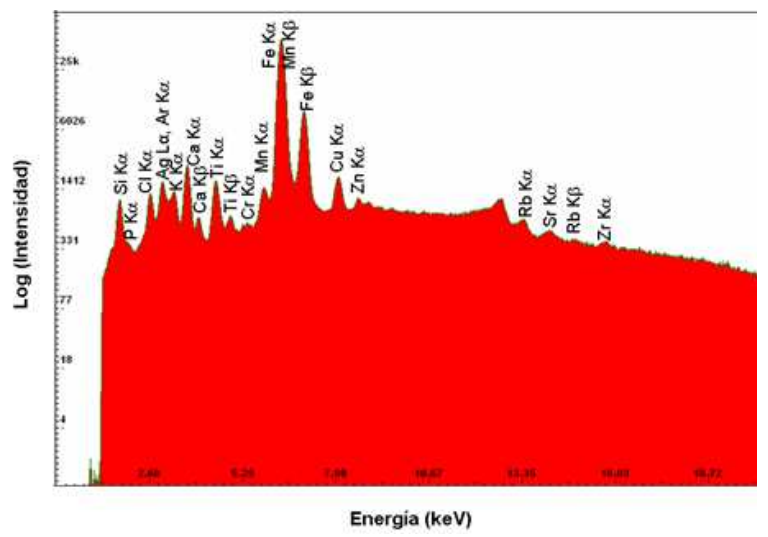

Figura 1: Espectro de fluorescencia de rayos $\mathrm{X}$ de la fracción arcillosa de la muestra M1.

También se aprecia un pico en la posición $\approx 12.8$ $\mathrm{keV}$, el cual corresponde a un caso de coincidecia, es decir, en que el detector recibe dos rayos $\mathrm{X}$ de $\mathrm{Fe} \mathrm{K}_{\alpha}$ $(6.398 \mathrm{keV})$ en forma casi simultánea; de tal manera que los detecta como si fuera un solo rayo $\mathrm{X}$ pero de doble energía. Además, se observa un pico alrededor de $3 \mathrm{keV}$, que es producido por los rayos X de Ar K emitidos por el aire irradiado por la fuente y en mayor proporción por los rayos X L de Ag emitidos por el tubo de rayos $\mathrm{X}$ utilizado y dispersados por la muestra.

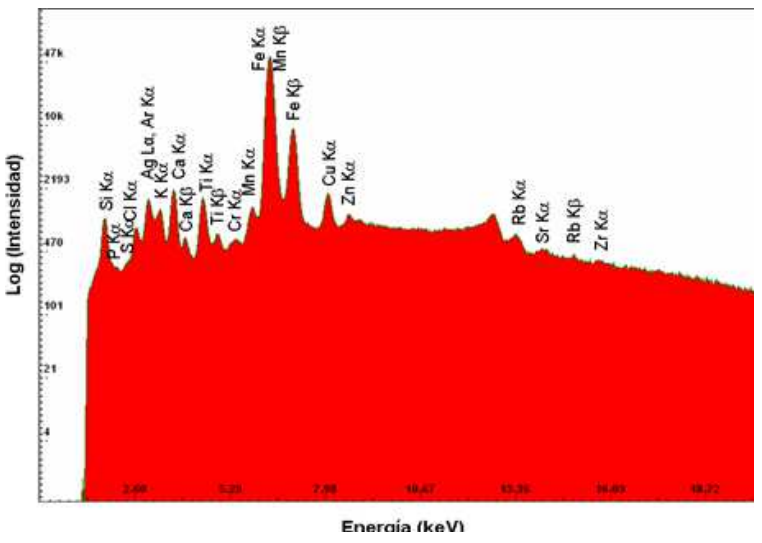

Figura 2: Espectro de fluorescencia de rayos X de la fracción arcillosa de la muestra M2.

\begin{tabular}{lcc}
\hline & M1 & M2 \\
\hline E. Q. & C $(\mathrm{mg} / \mathrm{kg})$ & $\mathrm{C}(\mathrm{mg} / \mathrm{kg})$ \\
\hline $\mathrm{Si}$ & 296,600 & 240,000 \\
$\mathrm{P}$ & 170 & 160 \\
$\mathrm{~S}$ & & 810 \\
$\mathrm{Cl}$ & 18,000 & 10,000 \\
$\mathrm{~K}$ & 5,000 & 5,000 \\
$\mathrm{Ca}$ & 8,500 & 5,800 \\
$\mathrm{Ti}$ & 2,600 & 2,500 \\
$\mathrm{Cr}$ & 120 & 100 \\
$\mathrm{Mn}$ & 700 & 600 \\
$\mathrm{Fe}$ & 38,500 & 39,900 \\
$\mathrm{Cu}$ & 750 & 600 \\
$\mathrm{Zn}$ & 150 & 150 \\
$\mathrm{Rb}$ & 180 & 180 \\
$\mathrm{Sr}$ & 250 & 200 \\
$\mathrm{Zr}$ & 30 & 30 \\
\hline
\end{tabular}

Tabla 3: Concentraciones de elementos químicos en la muestra M1 y M2, respectivamente. E.Q.: Elemento químico, C: Concentración.

\section{Resultados cuantitativos}

Para este fin fue necesario tener presente que el suelo está formado por un conjunto de elementos, entre ellos $\mathrm{H}, \mathrm{C}, \mathrm{N}, \mathrm{O}, \mathrm{Na}$ y Mg, que constituyen alrededor del $60 \%$ de la fracción arcillosa del suelo, y que no producen rayos X característicos detectables por la técnica utilizada pero que si dispersan rayos X y contribuyen a la parte continua del espectro de la FRXED. Por tal motivo, la matriz usada en el análisis del espectro debe considerar la presencia de estos elementos químicos. Los conteos registrados para la acumulación de los espectros de M1 y M2 fueron 2'010,745 y 2'012,928 cuentas, respectivamente. En la tabla 3 se muestran las concentraciones 
en $\mathrm{mg} / \mathrm{kg}$ de los elementos identificados en M1 y M2, existiendo aproximadamente las mismas concentraciones para ambas muestras. Los resultados cuantitativos permiten inferir que los metales pesados como $\mathrm{Cu}, \mathrm{Zn}$, $\mathrm{Rb}$, Sr y Zr, presentes en M1 y M2 no son tóxicos para las plantas, por cuanto se presentan en bajas concentraciones. Es importante mencionar que la concentración determinada para el silicio no es muy confiable, debido a que sus rayos $\mathrm{X}$ característicos poseen baja energía y pueden ser atenuados por el aire y la ventana del detector.

\section{Análisis por difractometría de rayos $\mathrm{X}$}

La Figura 3 muestra los difractogramas de las dos muestras estudiadas, obtenidos mediante el método de montaje de polvo. Las fases mineralógicas identificadas mediante este método son cuarzo, albita -en baja concentración-, y los minerales de arcilla montmorillonita e illita (tipo 2:1). Se observa la superposición de los picos de cuarzo y de illita en el pico de reflexión a
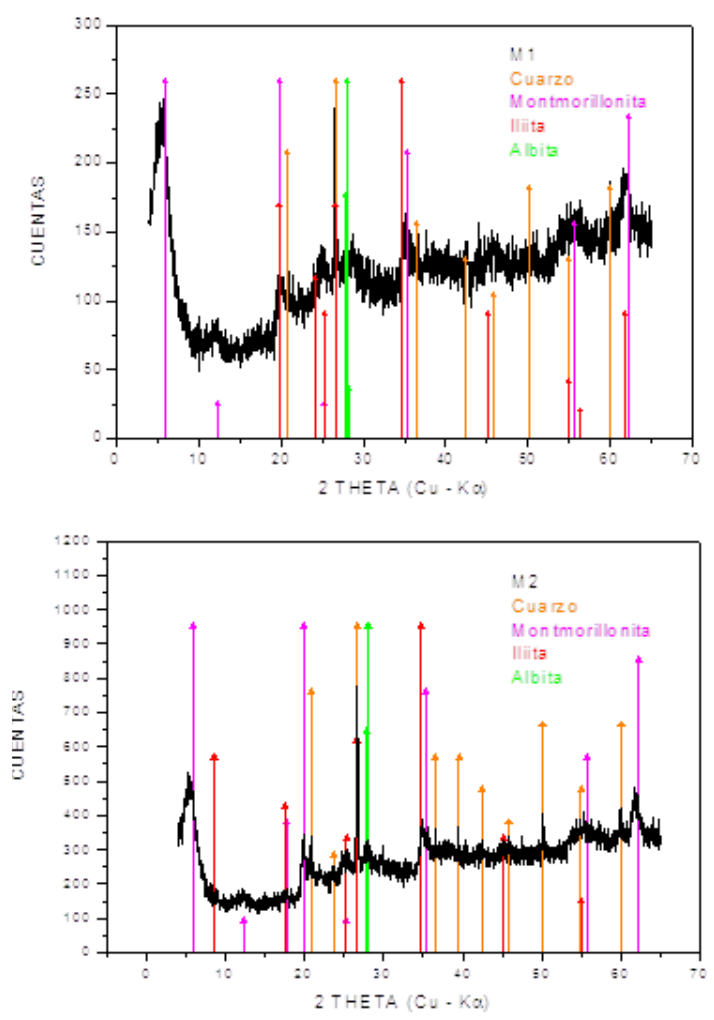

Figura 3: Difractogramas correspondientes a la fracción arcilla de las muestras M1 y M2, montadas por el método en polvo. $26.6^{\circ}$

En la Figura 4 se muestran los difractogramas correspondientes a las muestras M1 y M2, obtenidos mediante los métodos de Millipore y de Glass Slide, respectivamente. Por comparación, se observa que los pi$\cos (00 \ell)$, correspondientes a los minerales de arcilla, han incrementado notablemente su intensidad. Mediante estos métodos se identifica la presencia del mineral de arcilla caolinita (tipo 1:1), en adición a los minerales de arcilla montmorillonita e illita, identificados por el primer método; observándose la aparición de los picos (001) y (002) de la caolinita. Se debe notar que el pico (002) de la montmorillonita se solapa con el pico (001) de la caolinita.

Además, comparando el pico (001) de la illita de ambas muestras, se observa que en M1 dicho pico esta mejor resuelto que en M2. Esto se debe a que en M2 el pico (001) de la montmorillonita, que esta adyacente al pico (001) de la illita, es más intenso que en M1, lo cual no permite que el pico (001) de la illita se defina mejor.
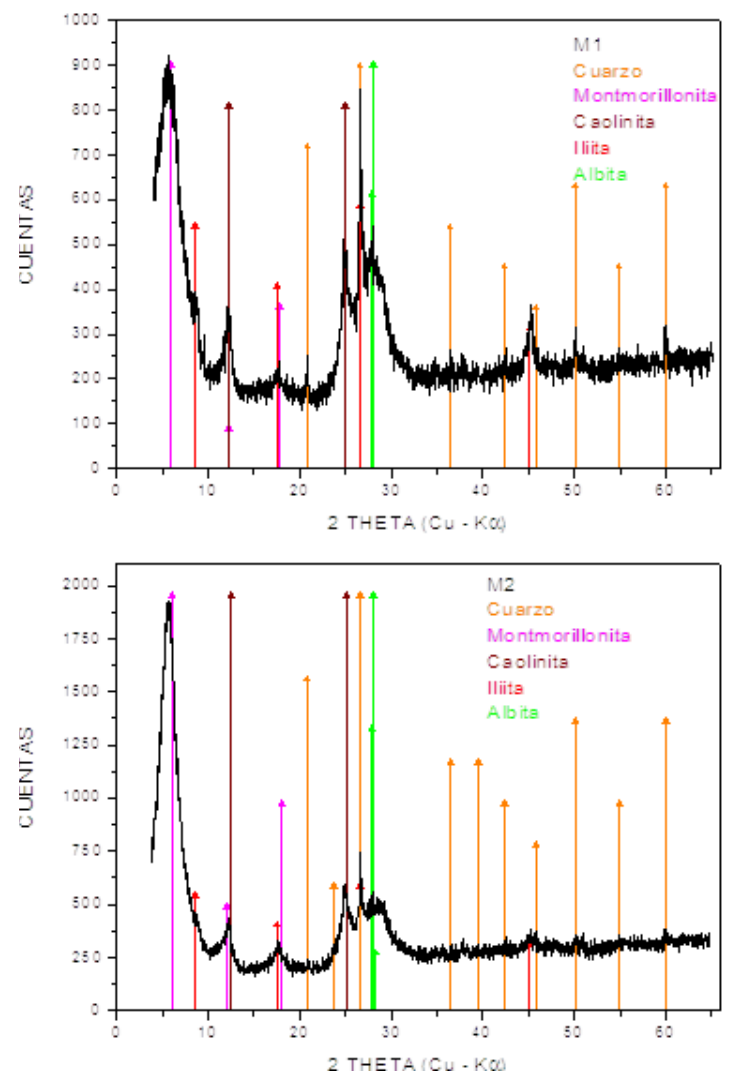

Figura 4: Difractogramas correspondientes a la fracción arcillosa de las muestras M1 y M2, montadas por los métodos de Millipore y Glass Slide respectivamente. Notar la superposición del pico de Caolinita (001) y Montmorillonita (002). 
Adicionalmente, se observa que el pico correspondiente a la posición $2 \theta$ de $26.6^{\circ}$, donde hay superposición de los picos (003) de illita y (101) de cuarzo, es más intenso en M1. Esto se debe a que el cuarzo en M2 se encuentra al fondo de la muestra, y es debido al proceso de sedimentación que se realiza en el método de Glass Slide, que se utilizó para montar el analito de M2; de manera que la contribución del cuarzo en dicha posición puede ser menor en comparación a su contribución en la misma posición en M1. Algo similar se puede decir del pico de reflexión en $45.2^{\circ} 2 \theta$, el cual recibe la contribución de los planos (005) de la illita y (201) del cuarzo.
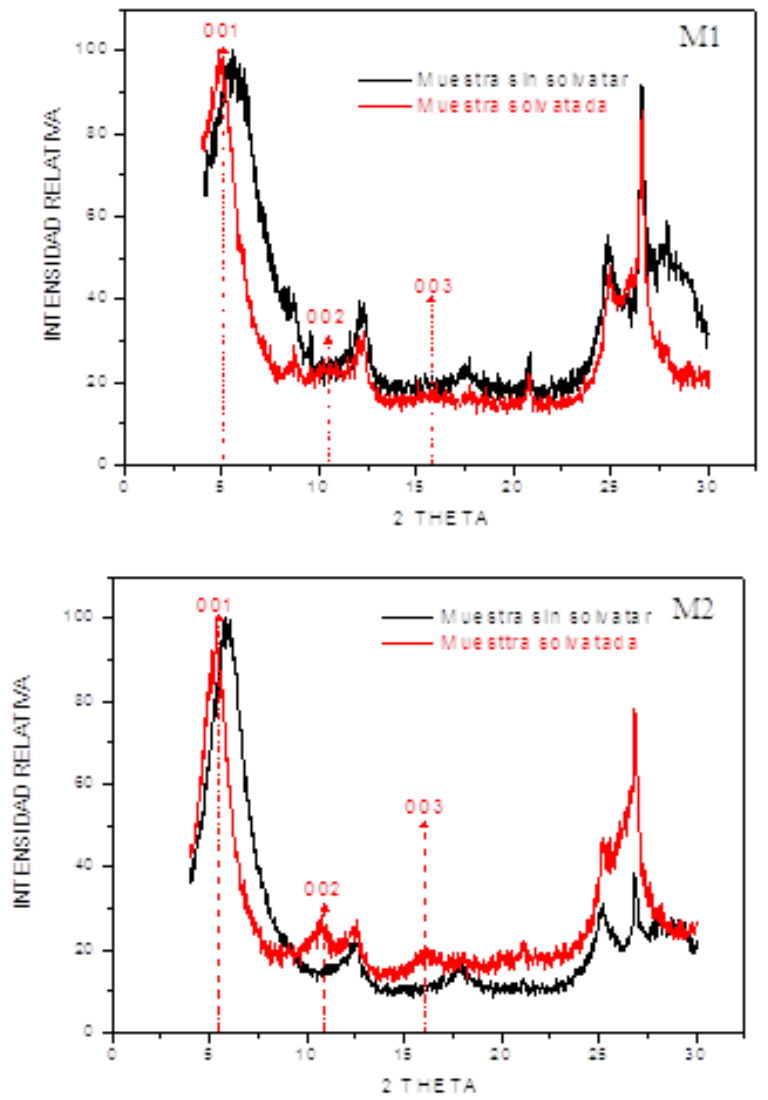

Figura 5: Superposición de los difractogramas de la fracción arcillosa de las muestras M1 y M2, con solvatación y sin solvatar. Se indican los picos (001), (002) y (003) de la montmorillonita desplazados a distancias interplanares mayores.

La Figura 5 muestra los difractogramas de las muestras M1 y M2 después que fueron tratadas con la técnica de solvatación con glicol de etileno. Se logra observar la expansión sufrida por la montmorillonita; que se manifiesta en los difractogramas obtenidos, por el corrimiento de los picos (001), (002) y (003) de dicho mineral a ángulos menores, esto es, a distancias interplanares mayores; lo cual demuestra la presencia de este mineral en ambas muestras. El corrimiento del pico (001) que se midió en el presente trabajo es aproximadamente igual a $1.99 \AA$ para M1 y $1.41 \AA$ para M2. El corrimiento del pico (002) de la montmorillonita a distancias interplanares mayores hace posible observar la contribución del pico (001) de la caolinita en dicha posición de manera más clara.

\section{Análisis por espectroscopia Mössbauer de transmisión}

Los resultados obtenidos mediante esta técnica indican que las muestras estudiadas son similares en su composición mineralógica. En las fracciones arcillosas de ambas muestras se observa aproximadamente la misma concentración de hierro, por cuanto poseen similares áreas de absorción resonante totales.
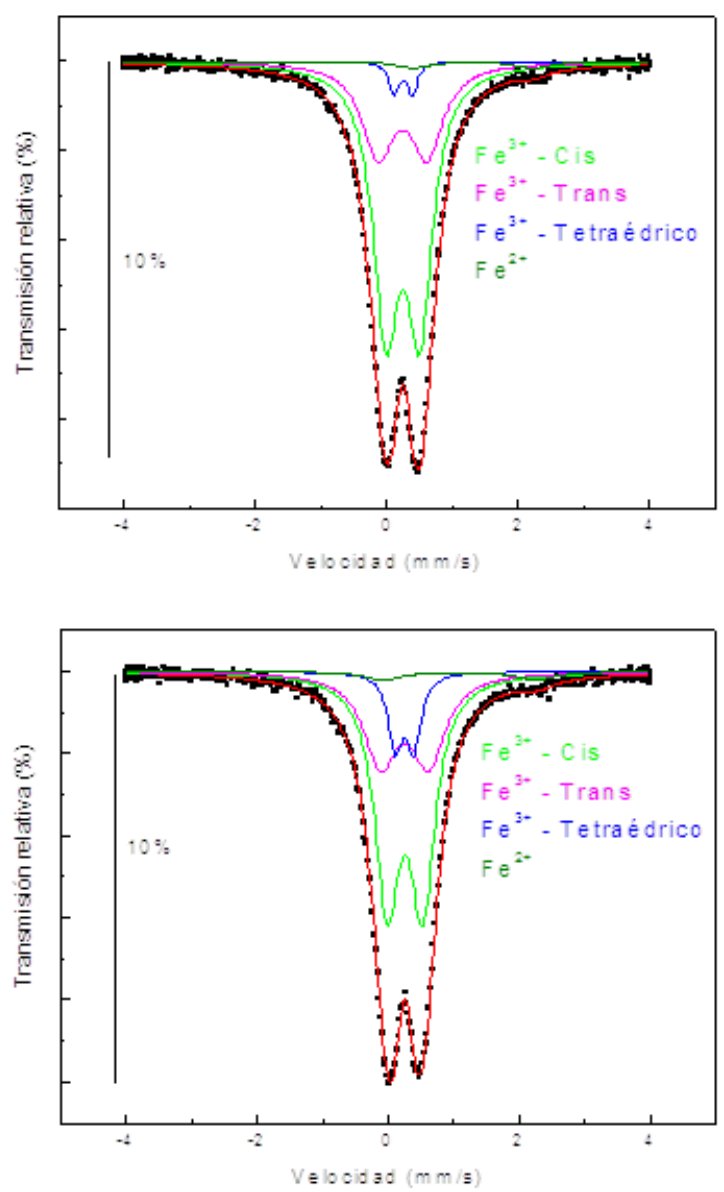

Figura 6: Espectro Mössbauer a baja velocidad de la fracción arcillosa de M1 y M2, respectivamente; sin tratamiento y a temperatura ambiente 
En la Figura 6 se muestran los espectros Mössbauer obtenidos a baja velocidad correspondientes a la fracción arcillosa de las muestras M1 y M2 respectivamente. Ambos espectros muestran en su parte central una resonancia de $\mathrm{Fe}^{3+}$. Estos espectros fueron ajustados con tres dobletes de $\mathrm{Fe}^{3+}$ y un doblete de $\mathrm{Fe}^{2+}$ bastante ancho. Para lograr convergencia en el ajuste de estos espectros, los valores de los anchos de línea de dos de los tres sitios de $\mathrm{Fe}^{3+}$ fueron fijados. Estos dos dobletes fueron asociados a cationes de $\mathrm{Fe}^{3+}$ ocupando sitios octaedrales cis y trans en la montmorillonita, por cuanto poseen aproximadamente el mismo valor del corrimiento isomérico y sus áreas de absorción resonante están en la relación de 2 a 1, respectivamente. El tercer doblete de $\mathrm{Fe}^{3+}$ posee un área mucho menor y fue asignado a cationes de $\mathrm{Fe}^{3+}$ en coordinación tetraedral en la montmorillonita, por tener un corrimiento isomérico y un desdoblamiento cuadrupolar pequeños. Por su lado, el sitio de $\mathrm{Fe}^{2+}$ no fue asignado a ningún mineral, ya que sus parámetros se solapan con aquellos de la montmorillonita, la illita y la caolinita. Además, el ensanchamiento observado en los picos de este doblete es un indicativo de un grado considerable de desorden estructural del entorno de este sitio de $\mathrm{Fe}^{2+}$; probablemente se encuentra formando parte de una fase no muy bien cristalizada.

Con la finalidad de apreciar si hay presencia de óxidos de hierro magnéticos, se tomaron espectros a alta velocidad de ambas muestras, los cuales se muestran en la Figura 7. En estos espectros no se observan fases magnéticas cristalinas.
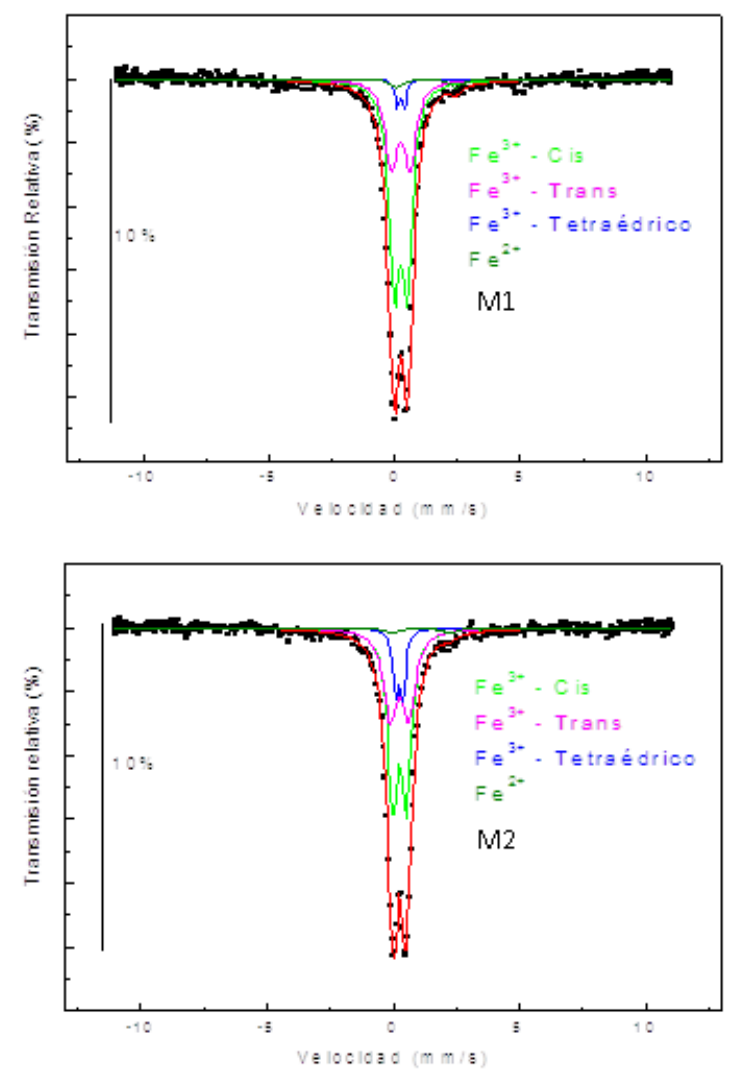

Figura 7: Espectros Mössbauer a alta velocidad de las fracciones arcillosas de M1 y M2, respectivamente; sin tratamiento a temperatura de ambiente.

\begin{tabular}{lccccc}
\hline Muestra & $\begin{array}{c}\text { Parámetro } \\
\text { hiperfino }\end{array}$ & $\begin{array}{c}\mathrm{Fe}^{3+}-\mathrm{Cis} \\
(\mathrm{mm} / \mathrm{s})\end{array}$ & $\begin{array}{c}\mathrm{Fe}^{3+} \text {-Trans } \\
(\mathrm{mm} / \mathrm{s})\end{array}$ & $\begin{array}{c}\mathrm{Fe}^{3+} \text {-Tetraédrico } \\
(\mathrm{mm} / \mathrm{s})\end{array}$ & $\begin{array}{c}\mathrm{Fe}^{2+} \\
(\mathrm{mm} / \mathrm{s})\end{array}$ \\
\hline $\mathrm{M} 1$ & & & & \\
\hline & ISO & 0.248 & 0.247 & 0.253 & 1.320 \\
& QUA & 0.510 & 0.745 & 0.276 & 1.801 \\
\hline $\mathrm{M} 2$ & & & & \\
\hline & ISO & 0.256 & 0.252 & 0.251 & 1.083 \\
& QUA & 0.547 & 0.742 & 0.283 & 2.325 \\
\hline
\end{tabular}

Tabla 4: Parámetros hiperfinos de la fracción arcillosa de las muestras M1 y M2.

En las tablas 4 y 5 se indican los parámetros hiperfinos y los porcentajes de área de absorción resonante para las muestras M1 y M2, respectivamente. Se observa que el área de absorción resonante total de las muestras M1 y M2 son iguales. En ambas muestras se aprecia que el porcentaje de absorción del sitio de $\mathrm{Fe}^{3+}$. Cis es mucho mayor que los porcentajes de absorción de los demás sitios y que el menor porcentaje de absorción corresponde al sitio de $\mathrm{Fe}^{2+}$. Además, se aprecia que las áreas de absorción resonante de los sitios de $\mathrm{Fe}^{3+}$. tetraédrico y de $\mathrm{Fe}^{2+}$ en M2 son mayores que las correspondientes a M1, lo que permite inferir que en los sitios de $\mathrm{Fe}^{3+}$-tetraédrico y de $\mathrm{Fe}^{2+}$ de $\mathrm{M} 2$ existen una mayor concentración de hierro que en los correspondientes de M1. 


\begin{tabular}{cccc}
\hline Muestra & $\begin{array}{c}\text { Sitio } \\
\text { Estructural }\end{array}$ & $\begin{array}{c}\text { Área de Absorción } \\
\text { Resonante }(\mathrm{mm} / \mathrm{s})\end{array}$ & $\begin{array}{c}\text { Porcentaje } \\
\text { de Absorción (\%) }\end{array}$ \\
\hline $\mathrm{M} 1$ & & \\
\hline & & 0.0822 & 65.22 \\
& $\mathrm{Fe}^{3+}$-Cis & 0.0371 & 29.33 \\
$\mathrm{Fe}^{3+}$-Trans & 0.0039 & 3.21 \\
$\mathrm{Fe}^{3+}$-Tetraédrico & 0.0030 & 2.24 \\
$\mathrm{Fe}^{2+}$ & 0.1262 & 100 \\
\hline Total & & \\
\hline $\mathrm{M} 2$ & & 0.0776 & 36.16 \\
& & 0.0415 & 10.40 \\
& $\mathrm{Fe}^{3+}$-Cis & 0.0143 & 3.38 \\
$\mathrm{Fe}^{3+}$-Trans & 0.0046 & 100 \\
\hline $\mathrm{Fe}^{3+}$-Tetraedrico & 0.1380 & \\
& $\mathrm{Fe}^{2+}$ & Total &
\end{tabular}

Tabla 5: Áreas de absorción resonante correspondientes a las muestras M1 y M2.

\section{Conclusiones}

Los resultados obtenidos permiten postular que las muestras estudiadas son de composición mineralógica y elemental similares, por cuanto están constituidas por los mismos minerales arcillosos y por casi los mismos elementos químicos. Los resultados cualitativos obtenidos por FRXED nos brindó información sobre el contenido de los cationes interlaminares, como $\mathrm{Ca}, \mathrm{Cl}$ y $\mathrm{K}$, y de sustitución, como Mn, Ti y Fe; esto es importante porque nos ayudó en la interpretación de los resultados obtenidos por DRX. El análisis cuantitativo de los resultados obtenidos por FRXED indica que ambas muestras poseen aproximadamente la misma concentración de hierro, aproximadamente $3.85 \%$ y $3.99 \%$ para M1 y M2, respectivamente.

Por otro lado, se ha demostrado la necesidad de utilizar los métodos de Millipore y de Glass Slide con el objetivo de obtener una mejor orientación de los cristales de arcilla. Se observó que el método de Millipore brinda información más real, sobre la concentración de minerales en las muestras estudiadas, que el método de glass slide. Además, se muestra la bondad de la técnica de solvatación con glicol de etileno para verificar la presencia de la montmorillonita.

Así mismo, los resultados obtenidos por la DRX y la EM muestran que el principal constituyente de la fracción arcillosa es la montmorillonita y que la mayor concentración de hierro se encuentra distribuida en los sitios octaédricos y tetraédricos de este mineral, ya que su área relativa de absorción resonante en los espectros Mössbauer es la mayor. Dicho mineral influye directamente en el intercambio de cationes aprovechables por los cultivos, por cuanto posee una elevada capacidad de intercambio catiónico.

\section{Referencias}

[1] Dorothy Carrol, Clay Minerals: A guide to their Xray identification, The Geological Society of America, Special paper 126 (1969).

[2] Eduardo Besoani, Mineralogía de Arcillas y Suelos, IICA (1985).

[3] Juan Davalos Prado, Espectroscopia Mössbauer, Fundamentos y Aplicaciones, Notas de Curso, Universidad Nacional de Ingeniería, agosto (1996).

[4] T. C. Gibb, Principles of Mössbauer Spectroscopy, Chapman and Hall, Londres (1976).
[5] Peter W. Birkeland, Soils and Geomorphology, 3ra Edición, Oxford University Press, Londres (1999).

[6] Manual de Laboratorio de Análisis de Suelos, Facultad de Ciencias Físicas, Universidad Nacional Mayor de San Marcos, Lima (2008)

[7] Ricardo Honorato Pinto, Manual de Edafología, 4ta Edición, Alfaomega, México (2000).

[8] R. A. Brand, NORMOS Mössbauer Fitting Program (1995). 